\title{
Estimación de los parámetros del modelo de un solo diodo del módulo fotovoltaico aplicando el método de optimización basado en búsqueda de patrones mejorado ${ }^{*}$
}

\author{
Andrés Felipe Tobón Mejía** \\ Jhon Jairo Rojas Montano*** \\ Sergio Ignacio Serna Garces ${ }^{* * *}$ \\ Jorge Aurelio Herrera Cuartas ${ }^{* * * *}$
}

Recibido: 27/11/2019 • Aceptado: 20/04/2020

https://doi.org/10.22395/rium.v20n38a1

\begin{abstract}
Resumen
En este artículo se propone el uso de un algoritmo de optimización basado en la búsqueda de patrones mejorada, aplicándolo a la estimación de los parámetros modelo de un diodo de una célula fotovoltaica. Los parámetros a estimar son corriente fotovoltaica, la saturación de corriente del diodo, la resistencia serie, la resistencia en paralelo y el factor de idealidad del diodo. La estimación es hecha a partir de los datos obtenidos de una curva conocida, es decir, que la celda fotovoltaica puede caracterizarse y los parámetros son extraídos de los datos de la curva. Los resultados son la identificación de los parámetros y la exactitud del modelo con respecto a la referencia en el punto de máxima potencia (MPP). Adicionalmente se realiza una comparación con el modelo obtenido con tres estimaciones realizadas con el algoritmo de optimización enjambre de partículas (PSO), bajo las mismas condiciones en cantidad de partículas y de iteraciones. El error encontrado muestra la similitud del modelo con la referencia obtenida de acuerdo con el algoritmo IPSM.
\end{abstract}

Palabras clave: electrónica de potencia; sistema fotovoltaico; MPPT; método de búsqueda de patrones; modelo de un solo diodo.

Artículo derivado de la investigación realizada en el Instituto Tecnológico Metropolitano (ITM) en el laboratorio de investigación electrónica y energías renovables.

** Magíster en Automatización y Control Industrial, Instituto Tecnológico Metropolitano; ingeniero en instrumentación y control industrial, Jaime Isaza Cadavid. Docente Ocasional, Instituto Tecnológico Metropolitano, Medellín, Colombia. Correo electrónico: andrestobon@itm.edu.co. Orcid: https://orcid.org/0000-0003-1254-231X

*** Magíster en Automatización y Control Industrial, Instituto Tecnológico Metropolitano; ingeniero electrónico, Instituto Tecnológico Metropolitano. Docente de cátedra, Instituto Tecnológico Metropolitano (ITM), Medellín, Colombia. Correo electrónico: jhonrojas7420@correo.itm.edu.co. Orcid: https://orcid.org/0000-0002-9573-9179

***** Doctor en Ingeniería Automática, Universidad Nacional de Colombia; magíster en Ingeniería, Universidad Nacional de Colombia; ingeniero electrónico, Universidad de Antioquia. Docente, Instituto Tecnológico Metropolitano (ITM), Medellín, Colombia. Correo electrónico: sergioserna@itm.edu.co. Orcid: https://orcid. org/0000-0002-1498-7323

****** Doctor en Informática Industrial, Universidad Autónoma de Barcelona; magíster en Informática Industrial, Universidad Autonoma de Barcelona; ingeniero electrónico, Universidad del Quindío. Decano, Universidad de Bogotá Jorge Tadeo Lozano, Bogotá, Colombia. Correo electrónico: jorgea.herrerac@utadeo.edu.co. Orcid: https://orcid.org/0000-0003-0273-4043 


\title{
Parameters Estimation of the Single Diode Model of a Photovoltaic Module Based on the Improved Patterns Search Method
}

\begin{abstract}
In this article, we propose the use of the optimization algorithm based on improved pattern search (IPSM), applying it to the estimation of the model parameters of a single diode of a photovoltaic cell. The parameters to be estimated are the photovoltaic current, the saturation current of the diode, the series resistance, the resistance in parallel, and the ideality factor of the diode. The estimation is made from the data obtained from a known curve, that is to say, that a photovoltaic cell could be characterized and from the data of the curve the parameters are extracted. The results are the identification of the parameters and the accuracy of the model concerning the reference at the point of maximum power (MPP). Additionally, a comparison is made with the model obtained with three estimations made with the particle swarm optimization algorithm (PSO), under the same conditions in the number of particles and iterations. The error found shows the similarity of the model with the reference obtained using the IPSM algorithm.
\end{abstract}

Keywords: power electronic; photovoltaic system; MPPT; pattern search method; single-diode model. 


\section{INTRODUCCIÓN}

Ante la creciente demanda de energía y el deterioro ambiental, los gobiernos están promoviendo el uso de energías renovables no convencionales donde antes se empleaban combustibles fósiles [1]. La producción de energía a partir de la luz del sol es, en la actualidad, la fuente renovable de mayor uso a nivel mundial [2], de ahí la necesidad de desarrollar celdas solares más eficientes, confiables, de bajo costo y que permitan maximizar la transformación de energía bajo cualquier condición ambiental. La celda fotovoltaica (PV) es el dispositivo básico para la generación de energía eléctrica a partir de fotones. A pesar de los avances tecnológicos que se han tenido en su fabricación, la eficiencia de esta está limitada por factores como radiación solar, temperatura y sombreado [3-5].

Por múltiples propósitos [6-8], una celda PV puede ser modelada con diferentes niveles de exactitud y complejidad [9-13]. Los modelos circuitales de uno y dos diodos son los más utilizados en la literatura: el de un diodo por su simplicidad, ya que el número de parámetros por estimar es reducido; y el de dos diodos por su exactitud, ya que es una mejor representación del comportamiento de la celda en condiciones de baja radiación solar [14-15].

Con el objetivo de poder evaluar el desempeño de una celda PV, controlarla, conocer su eficiencia y establecer su punto de máxima potencia (Maximum Power Point Tracking [MPPT]), es necesario encontrar los parámetros del modelo de la celda. Se han realizado múltiples trabajos con diferentes enfoques para tratar de estimar los parámetros del modelo de la celda $\mathrm{PV}$, los cuales pueden ser clasificados en tres categorías: métodos analíticos, métodos de optimización y métodos híbridos. Estos últimos utilizan enfoques analíticos en combinación con algoritmos de optimización metaheurísticos [16].

En [17-24] se emplean métodos analíticos para estimar los parámetros del modelo de un solo diodo de una celda PV; en algunos casos el modelo empleado solo considera pérdidas óhmicas y en otros se introducen, además, las corrientes de fuga en la juntura del semiconductor.

En [25-26] se han estimado los parámetros del modelo de la celda solar de uno y dos diodos empleando el algoritmo de optimización basado en un enjambre de abejas artificial. La función objetivo está basada en el error cuadrático medio del error de los datos experimentales y los estimados. Los algoritmos de evolución diferencial son empleados en [27-29] con el objetivo de estimar los parámetros de los modelos de la celda fotovoltaicas, tanto de un diodo como de dos diodos. En todos los casos, la función objetivo es reiterativa: el error cuadrático medio entre los datos experimentales y los 
estimados. PSO (Particle Swarm Optimization) es otro algoritmo de optimización metaheurístico empleado para estimar los parámetros del modelo de dos y tres diodos de la celda fotovoltaica [30], en el cual la función objetivo es el error absoluto promedio de la corriente del panel. En [31-32] la sumatoria del error absoluto individual de los cinco parámetros del modelo de un diodo de la celda fotovoltaica es considerada como la función objetivo para la estimación a partir del algoritmo de búsqueda de patrones. Las ventajas de este algoritmo con respecto a otros, como algoritmos no lineales basados en mínimos cuadrados, algoritmos basados en el método de Newton modificado y algoritmos genéticos, se evidencian en este artículo. Otros métodos de optimización se han empleado con el fin de estimar los parámetros del modelo de uno y dos diodos de la celda fotovoltaica, entre ellos están Biogeography-Based Optimization (BBO) [33], Innovative Global Harmony Search (IGHS) [34], Simulated Annealing (SA) [31], Bird Mating Optimization (BMO) [35], entre otros.

Este artículo está dividido en cinco partes. En primer lugar, se presenta el modelo de un diodo de la celda fotovoltaica y la solución explícita de la ecuación del modelo. En segundo lugar, se describe detalladamente del algoritmo de búsqueda de patrones mejorado (Improved Pattern Search Method [IPSM]). Posteriormente, se presenta el algoritmo de simulación de curvas $V-1$ de paneles comerciales. A continuación, se implementa el algoritmo IPSM y se compara con PSO. Finalmente, se presentan las conclusiones.

\section{MODELO DE UN SOLO DIODO DE LA CELDA FOTOVOLTAICA}

El modelado de PV tiene como uno de sus objetivos representar las curvas I-V de la celda o arreglo bajo diferentes condiciones de radiación solar o temperatura. Como se mencionó en la introducción, los modelos basados en un circuito eléctrico equivalente a uno y dos diodos son los más utilizados en la literatura; el primero por su simplicidad y el segundo por su exactitud.

En el modelo de dos diodos (descrito por la ecuación 1) la corriente del panel fotovoltaico $I_{p v}$ es debida a la corriente fotoinducida $I_{p h}$ menos las pérdidas debidas a la absorción de la luz solar $I_{d 1}$, al mecanismo de recombinación en la juntura del diodo $I_{d 2}$ y las pérdidas óhmicas que producen fugas de corriente $I_{R h}[36]$.

$$
I_{p v}=I_{p h}-I_{d 1}-I_{d 2}-I_{R h}
$$

Típicamente, el modelo de la celda fotovoltaica se ajusta para el caso de celdas de silicio cristalino, lo que implica despreciar las pérdidas debido a la recombinación, donde $I_{d 2}=0$ (véase Figura 1). Por lo tanto, el modelo de la celda fotovoltaica se convierte en el modelo de un diodo, el cual está dado en la ecuación 2 [36]. 


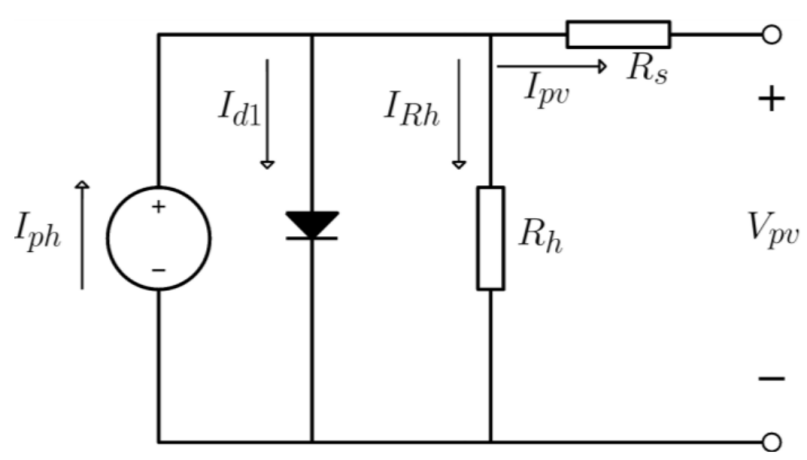

Figura 1. Circuito equivalente del modelo de un diodo de una celda fotovoltaica Fuente: elaboración propia.

$$
I_{p v}=I_{p h}-I_{d 1}\left[e^{\frac{V_{p v}+R_{s} I_{p v}}{\eta V_{t}}}-1\right]-\frac{V_{p v}+R_{s} I_{p v}}{R_{h}}
$$

En este caso, $I_{d 1}$ es la corriente de saturación del diodo, $\eta$ es el factor de idealidad del diodo, que para un diodo ideal es 1 . Sin embargo, típicamente $\eta \in[1,1,5], R_{s} \mathrm{y}$ $R_{h}$ son parámetros desconocidos y representan las pérdidas de voltaje y corriente del panel, $V_{t}=k T / q$ es el voltaje térmico de la juntura $P N$ de la celda fotovoltaica, y se calcula a partir de la temperatura $T$, la constante de Boltzmann $k$ y la carga del electrón $q$. Finalmente, $V_{p n}$ es el voltaje de salida del panel.

Dependiendo de la precisión deseada en la simulación, el modelo de un diodo puede simplificarse aún más. Por ejemplo, se utilizan también modelos con $R_{s}=0$, con $R_{h}=0$ o bien con $R_{s}=R_{h}=0$. Este circuito equivalente de un diodo para la celda fotovoltaica, es solo una simplificación de esta y no representa todas sus dinámicas.

\subsection{Solución explícita de La ecuación del modelo}

La ecuación 2 no admite una solución basada en funciones elementales, pero sí una solución explícita basada en la función Lambert W [37]. Reescribiendo la ecuación 2, obtenemos la ecuación 3 .

$$
e^{\left(\frac{R_{s} I_{p v}}{\eta V_{t}}+\frac{V_{p v}}{\eta V_{t}}\right)=-I_{p v} \frac{R_{s}+R_{h}}{R_{h} I_{d 1}}+\frac{\left(I_{p h}+I_{d 1}\right) R_{h}-V_{p v}}{R_{h} I_{d 1}}}
$$

Esta ecuación es de la forma dada en la ecuación 4.

$$
P^{a \cdot x+b}=c \cdot x+d
$$


Donde $P$ es la base de los logaritmos naturales, $x=I_{p v}, a=\frac{R_{s}}{\eta V_{t}}, b=\frac{V_{p v}}{\eta V_{t}}, c=\frac{R_{s}+R_{h}}{R_{h} I_{d 1}}$, $d=\frac{\left(I_{p h}+I_{d 1}\right) R_{h}-V_{p v}}{R_{h} I_{d 1}} \cdot$ Con la sustitución dada en la ecuación 5 , la ecuación 4 se convierte en la ecuación 6.

$$
\begin{aligned}
-t & =a \cdot x+\frac{a \cdot d}{c_{c} \cdot d} \\
t \cdot P^{t} & =-\frac{a}{c} P^{\left(b-\frac{a}{c}\right)}
\end{aligned}
$$

Por definición de la función trascendente Lambert's W obtenemos la ecuación 7 cuya solución está dada en la ecuación 8 .

$$
\begin{gathered}
t=\frac{W\left(-\frac{a}{c} P^{\left(b-\frac{a \cdot d}{c}\right)} \ln P\right)}{\ln P} \\
x=-\frac{1}{a \cdot \ln P} W\left(-\frac{a \cdot \ln P}{c} P^{\left(b-\frac{a \cdot d}{c}\right)}\right)-\frac{d}{c}
\end{gathered}
$$

Para el caso del modelo de un solo diodo de la celda fotovoltaica, la relación de $I_{p v}$ en función de $V_{p v}$, según la ecuación 8 , está dada en la ecuación 9.

$$
I_{p v}=\frac{\left(I_{p h}+I_{d 1}\right) R_{h}-V_{p v}}{R_{h}+R_{s}}-\frac{\eta V_{t}}{R_{s}} \cdot W\left(\frac{R_{s} R_{h} I_{d 1}}{\left(R_{h}+R_{s}\right) \eta V_{t}} e^{\left(\frac{V_{p v}}{\eta V_{t}}+\frac{\left(I_{p h}+I_{d 1}\right) R_{h}-V_{p v}}{\left(R_{s}+R_{h}\right) \eta V_{t}}\right)}\right)
$$

\section{ALGORITMO BÚSQUEDA DE PATRONES MEJORADO (IPSM)}

El IPSM es un algoritmo que divide el campo de búsqueda en un número de mallas igual al número de variables a optimizar, las cuales están previamente acotadas. El rango de cada variable a optimizar se divide en $n$ puntos equidistantes. El algoritmo (véase figura 2) busca los valores de las variables que optimizan la función de costo definida en la ecuación 10, FO donde Error $_{i}$ de la ecuación 11 es el error entre la corriente real $I_{p v, r e a l}$ dada por la curva experimental $V-I$ y la corriente calculada $I_{p v, c a l c}$ de acuerdo con la ecuación 9 o la ecuación 12. Finalmente, $N$ es el número de puntos de la curva experimental $V-I$. 


$$
\begin{gathered}
F O=\sqrt{\frac{\left(\text { Error }_{i}\right)^{2}}{N}} \\
\text { Error }_{i}=\sum_{k=1}^{N=\frac{V_{o c}}{\tilde{A} V}}\left(I_{p v, \text { real }}-I_{p v, \text { calc }}\right) \\
I_{p v, \text { calc }}=I_{p h}-I_{d 1}\left[e^{\frac{V_{p v}+R_{s} I_{p v, \text { real }}}{\eta V_{t}}}-1\right]-\frac{V_{p v}+R_{s} I_{p v, \text { real }}}{R_{h}}
\end{gathered}
$$

Luego de determinar los valores de las variables que optimizaron la función de costo, se establecen nuevas mallas cuyo rango estará entre los dos puntos adyacente a los valores hallados (véase figura 3). A modo de ejemplo, si las mallas grandes ( $\mathrm{Mg}$ ) están conformada por $n$ puntos equidistantes, entonces cada $i$-ésima variable está representada en la ecuación 13 .

$$
M g_{i}=\left[Q_{1} Q_{2} Q_{3} Q_{4} \ldots Q_{n}\right]
$$

donde:

$$
Q_{1}<Q_{2}<Q_{3}<Q_{4}<\ldots<Q_{n}
$$

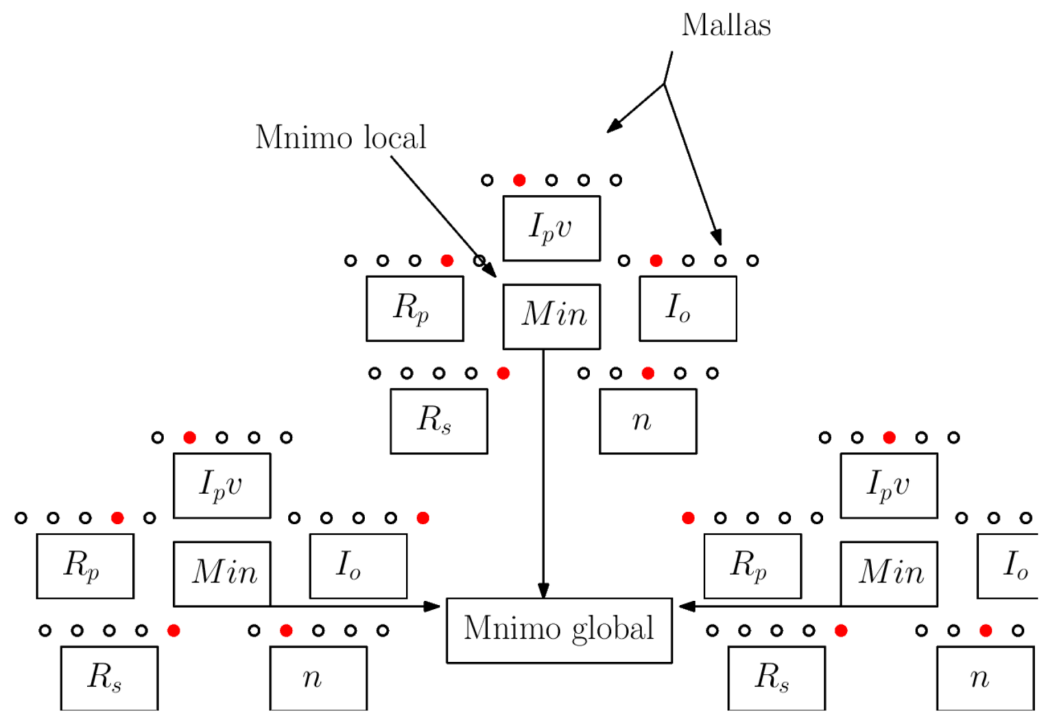

Figura 2. Procedimiento para la estimación de los parámetros del modelo matemático de un módulo fotovoltaico usando el algoritmo de optimización PSM 
Las mallas pequeñas $(\mathrm{Mp}$ ) estarán conformadas por $m$ puntos alrededor del punto de la malla grande en donde se encuentre el mínimo local que se halló previamente en la ecuación 15. Como muestra la ecuación 16, los puntos tendrán un orden de menor a mayor.

$$
M p_{i}=\left[q_{1} q_{2} q_{3} q_{4} \ldots q_{m}\right]
$$

donde:

$$
q_{1}<q_{2}<q_{3}<q_{4}<\ldots<q_{m}
$$

Como ejemplo, si el mínimo local hallado en la malla grande fue $Q_{2}$, entonces la malla pequeña debe cumplir lo establecido en la ecuación 17.

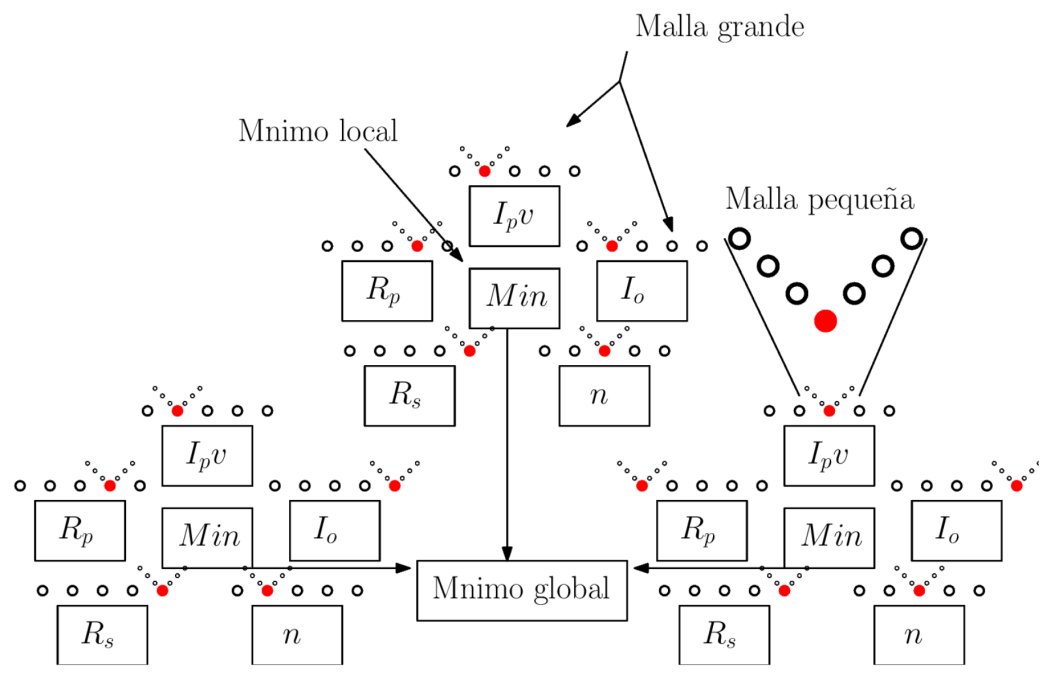

Figura 3. Gráfico del procedimiento para la estimación de los parámetros de modelo matemático de un módulo fotovoltaico usando el algoritmo de optimización IPSM Fuente: elaboración propia.

$$
M p_{i} \in\left[Q_{1} Q_{3}\right]
$$

Una vez que se definen las mallas grandes $(M g)$ y pequeñas $(M p)$ se procede a encuestar cada una de las variables de las mallas grandes y así obtener los valores que más se acercan al punto óptimo de la función de costo. Estos valores serán el centro de las mallas pequeñas. En este sentido, se realiza de nuevo una encuesta para lograr así una búsqueda exhaustiva (véase figura 3). 


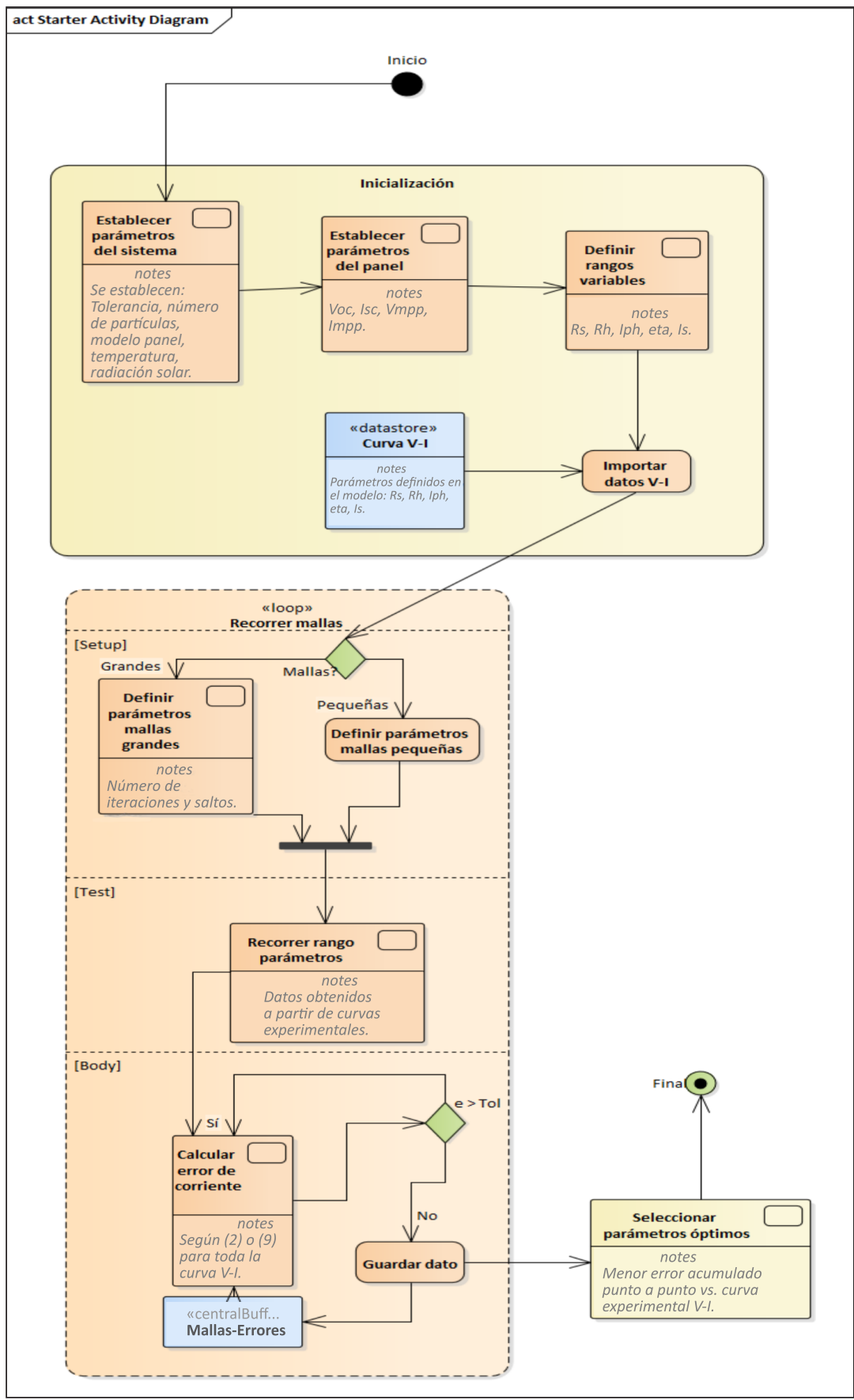

Figura 4. Diagrama de actividades de la optimización IPSM de los parámetros del modelo de la celda fotovoltaica

Fuente: elaboración propia. 
Si se encuentra un nuevo valor que esté aún más cerca del punto óptimo de la función de costo, este reemplazará al previamente hallado para dicha malla. Por cada iteración se reducen el rango de las nuevas mallas pequeñas, y el algoritmo finaliza después de determinado número de iteraciones o cuando el error acumulado sea menor que la tolerancia.

Para finalizar la búsqueda, se puede definir una tolerancia que garantice la exactitud deseada. Pero si esta exactitud no puede alcanzarse, el algoritmo quedará en un lazo infinito. Por lo tanto, se recomienda definir un número máximo de iteraciones que sacaría al algoritmo del posible bucle infinito, en caso de no alcanzar la exactitud esperada.

La figura 4 muestra el diagrama de actividades del algoritmo implementado en Matlab $^{\circledR}$ y su seudocódigo se presenta en la figura 5. El algoritmo tiene una actividad de inicialización en la cual se establecen los parámetros del sistema (tolerancia, número de partículas, modelo del panel, temperatura, radiación solar, etc.), los parámetros del panel (voltaje de circuito abierto, corriente de corto circuito y punto de operación de máxima potencia), el rango de los parámetros a optimizar (resistencia serie y paralela, corriente fotovoltaica, corriente inversa y factor de idealidad del diodo) y finalmente se importan los datos de la curva experimental $V-I$ del panel.

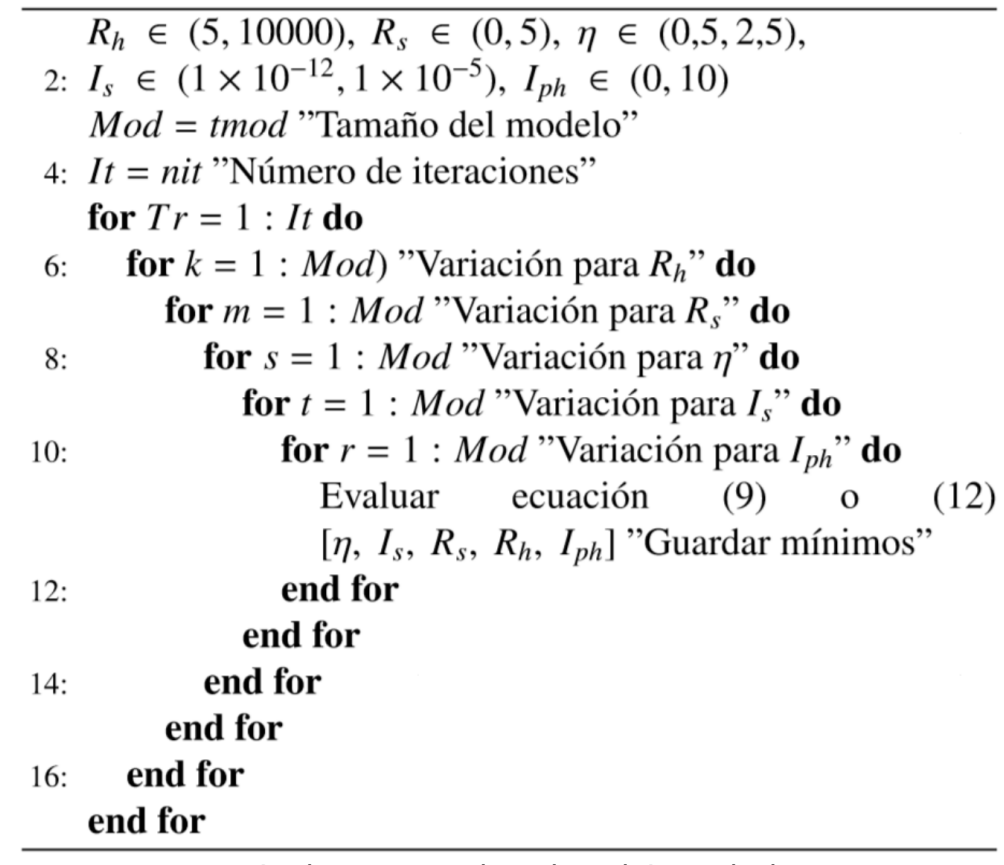

Figura 5. Método propuesto basado en búsqueda de patrones Fuente: elaboración propia. 
En la actividad estructurada del tipo loop se representan los ciclos anidados. Estos recorren los rangos previamente definidos en los cinco parámetros del modelo de un solo diodo de la celda fotovoltaica que se quieren optimizar en la ecuación 2, esto es, $R_{s}, R_{h}, I_{p h}, \eta, I_{d 1}$. Además, para cada valor de los cinco parámetros se recorre la curva experimental $V-I$ completa del panel, cuyo modelo se desea obtener. La actividad estructurada finaliza luego de recorrer el rango completo de los cinco parámetros del modelo, y la selección de los valores óptimos de dichos parámetros se da de acuerdo con una tolerancia definida establecida por la ecuación 11. Debe tenerse en cuenta que esta actividad estructurada sirve para modelar las consultas realizadas tanto a las mallas grandes como a las pequeñas.

Finalmente, luego de recorrer las mallas grandes y pequeñas, se obtienen los valores de los parámetros que optimizan el modelo de un diodo de la celda fotovoltaica con cuya curva experimental $V-I$ se cuenta.

El método de búsqueda de patrones mejorado (IPSM) se puede representar por el pseudocódigo presentado en la Figura 5.

\section{OBTENCIÓN DE LAS CURVAS EXPERIMENTALES DE LOS PANELES FOTOVOLTAICOS}

Para poder validar los resultados obtenidos luego del proceso de optimización basado en IPSM se generan los datos de las curvas del panel utilizando el algoritmo propuesto por [38], en donde se encuentran los parámetros de un modelo matemático equivalente. De esta manera se generan, finalmente, las curvas características. Para las pruebas experimentales se generaron las curvas $V-I$ de cuatro paneles con características diferentes, tal como puede verse en la tabla 1 .

Tabla 1. Paneles fotovoltaicos empleados para comparar la curva característica

\begin{tabular}{ccccc}
\hline Panel & Celdas & $P_{\max }(W)$ & $\left(V_{m p}, I_{m p}\right)$ & $I_{s c}(A)$ \\
\hline MSX 60 & 60 & 60 & $(17,1 \mathrm{~V}, 3,50 \mathrm{~A})$ & 3,80 \\
\hline JS65 & 36 & 65 & $(17,5 \mathrm{~V}, 3,71 \mathrm{~A})$ & 4,00 \\
\hline KC200GT & 54 & 200 & $(26,3 \mathrm{~V}, 7,61 \mathrm{~A})$ & 8,21 \\
\hline STP245S & 60 & 245 & $(30,5 \mathrm{~V}, 8,04 \mathrm{~A})$ & 8,52 \\
\hline
\end{tabular}

Fuente: elaboración propia.

En las figuras 6, 7, 8 y 9 pueden verse, en el lado izquierdo, la curva $V-P$ estimada y la experimental de los paneles BP MSX-6, JS65 Series, KC200GT y STP245S respectivamente. El lado derecho de estas figuras muestra un acercamiento al punto de máxima potencia, el cual es el más importante debido a la exigencia de extraer siempre la máxima potencia del panel. Se puede observar que la pérdida de potencia más alta entre el modelo estimado y el experimental se da para el panel JS65 Series, 
donde la diferencia está por debajo del 1,1\%. Para los demás paneles, la pérdida en potencia está por debajo del $1 \%$. En los demás sectores de la curva $V-P$ el algoritmo alcanza una aproximación muy cercana a la ideal.
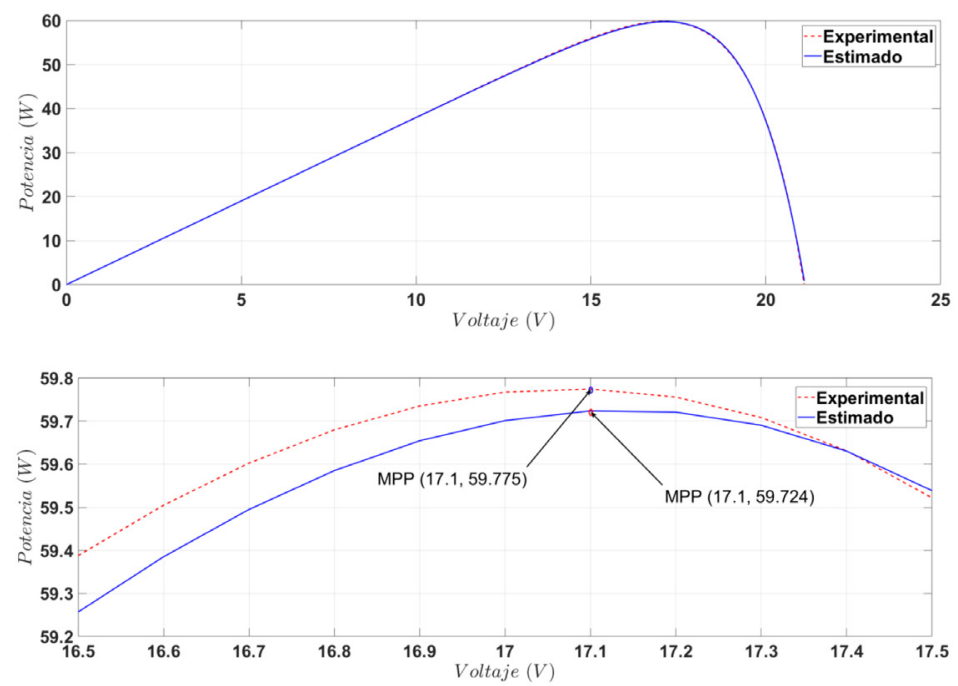

Figura 6. Curva experimental del BP MSX-60 vs. modelo matemático estimado según IPSM Fuente: elaboración propia.
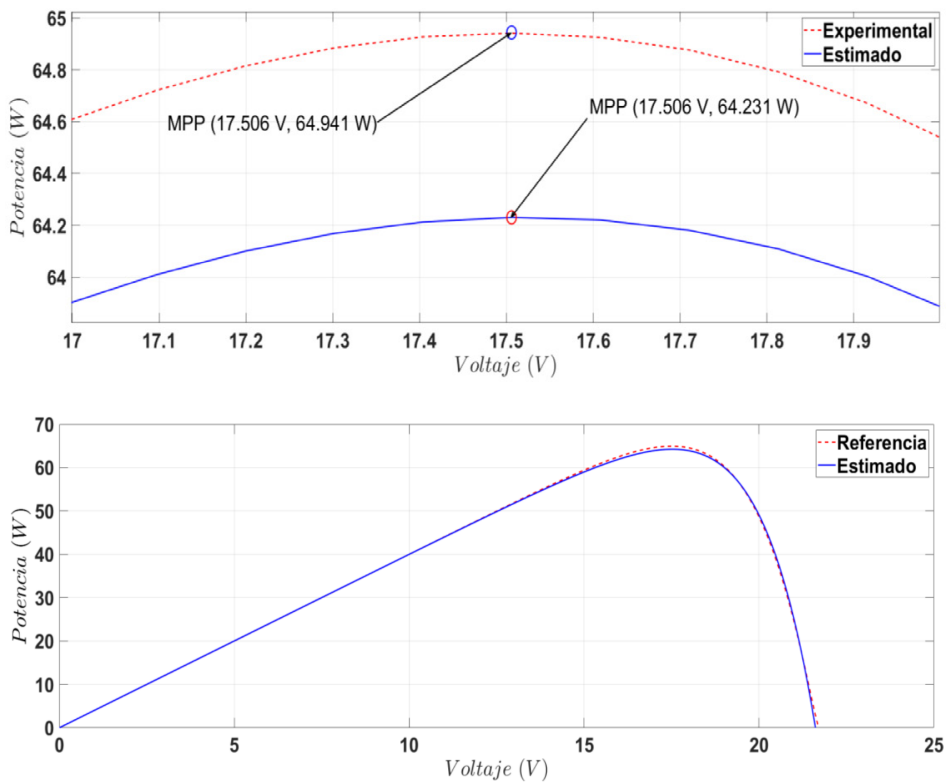

Figura 7. Curva experimental del panel JS65 vs. Modelo matemático estimado según IPSM Fuente: elaboración propia. 

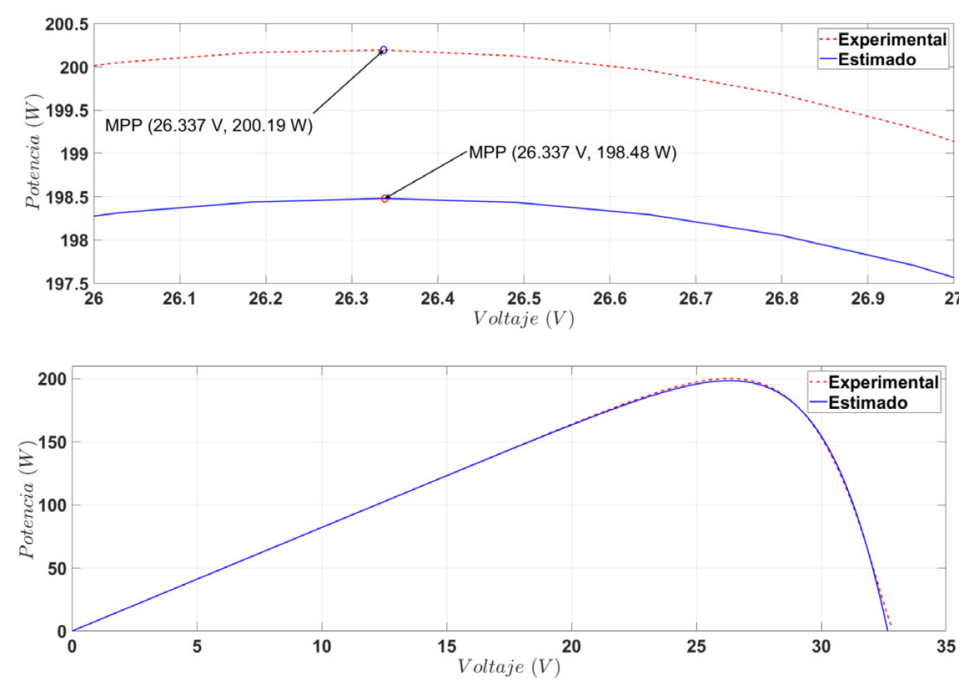

Figura 8. Curva experimental del panel KC200GT vs. modelo matemático estimado según IPSM Fuente: elaboración propia.
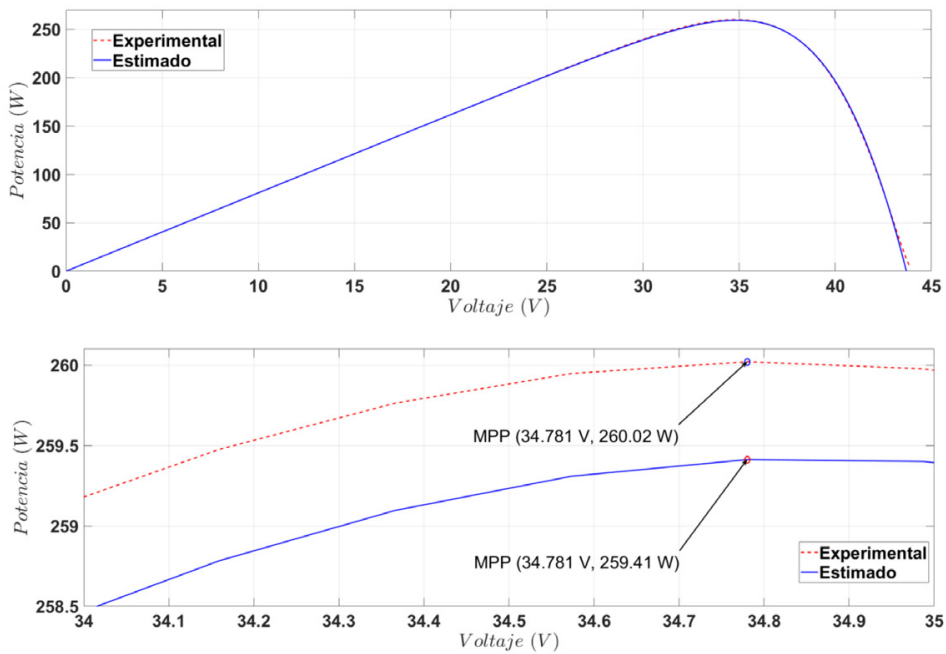

Figura 9. Curva experimental del panel STP245 vs. Modelo matemático estimado según IPSM Fuente: elaboración propia.

\section{COMPARACIÓN DEL ALGORITMO IPSM CON EL ALGORITMO PSO}

La validación del algoritmo de optimización IPSM para la estimación de los parámetros del modelo de un diodo del panel fotovoltaico se hizo bajo las mismas condiciones y la misma cantidad de partículas e iteraciones contra el algoritmo de optimización PSO [39]. 
Para la prueba se tomaron diez partículas con cincuenta iteraciones en ambos algoritmos de optimización (IPSM y PSO). También se aplicaron los algoritmos a la estimación de los cinco parámetros $\left(R_{h}, R_{s}, \eta, I_{s}, I_{p h}\right)$ sobre los paneles BP MSX-60, JS65 Series, KC200GT y STP245S de acuerdo con los rangos que se describen en la tabla 2.

Tabla 2. Rangos para la estimación de las variables $n, I_{d 1}, R_{s^{\prime}}, R_{h^{\prime}} I_{p h}[40]$

\begin{tabular}{ccc}
\hline Variables & Limite inferior & Limite superior \\
\hline$R_{h}(\Omega)$ & 5 & 10.000 \\
\hline$R_{s}(\Omega)$ & 0 & 5 \\
\hline$\eta$ & 0,5 & 2,5 \\
\hline$I_{d 1}(A)$ & $1 e^{-12}$ & $1 e^{-5}$ \\
\hline$I_{p h}(A)$ & 0 & 10 \\
\hline
\end{tabular}

Fuente: elaboración propia.

Los resultados se exponen en las figuras 10, 11, 12 y 13 para los paneles BP MSX60, JS65 Series, KC200GT y STP245S respectivamente. Las figuras muestran la curva experimental de cada uno de los paneles (línea azul continua), la optimización obtenida con IPSM (línea punteada roja) y tres aproximaciones obtenidas con PSO de diez partículas.

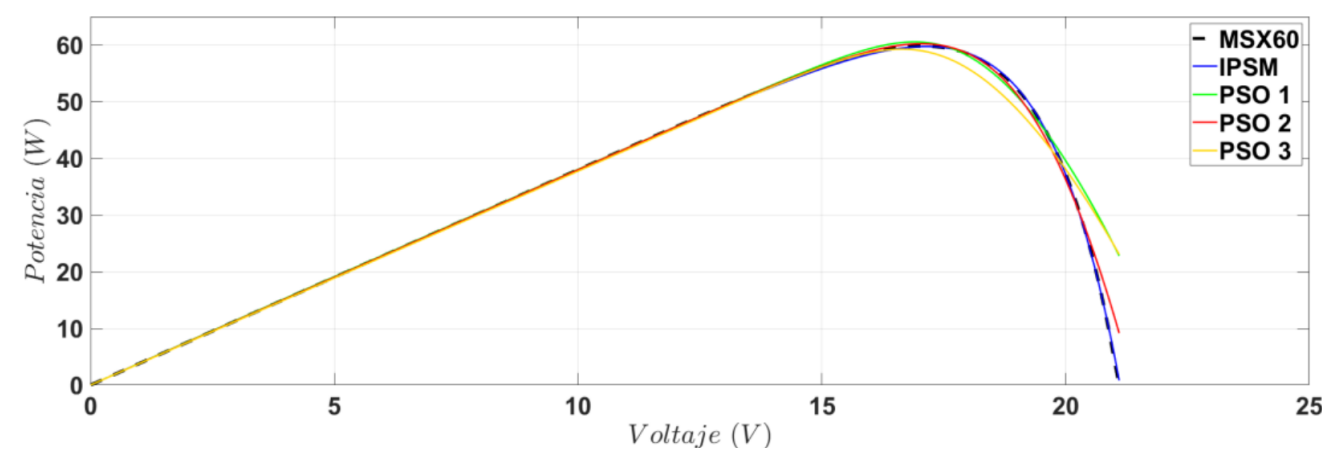

Figura 10. Comparación entre los datos de referencia del panel BP MSX60, la estimación según IPSM y tres estimaciones según PSO Fuente: elaboración propia. 


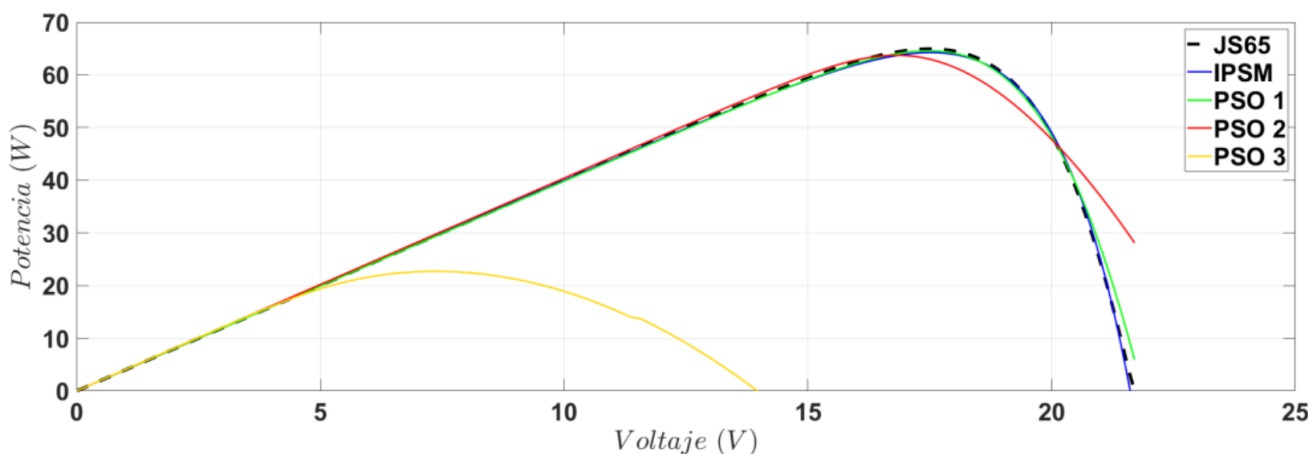

Figura 11. Comparación entre los datos de referencia del panel JS65 series, la estimación según IPSM y tres estimaciones según PSO Fuente: elaboración propia.

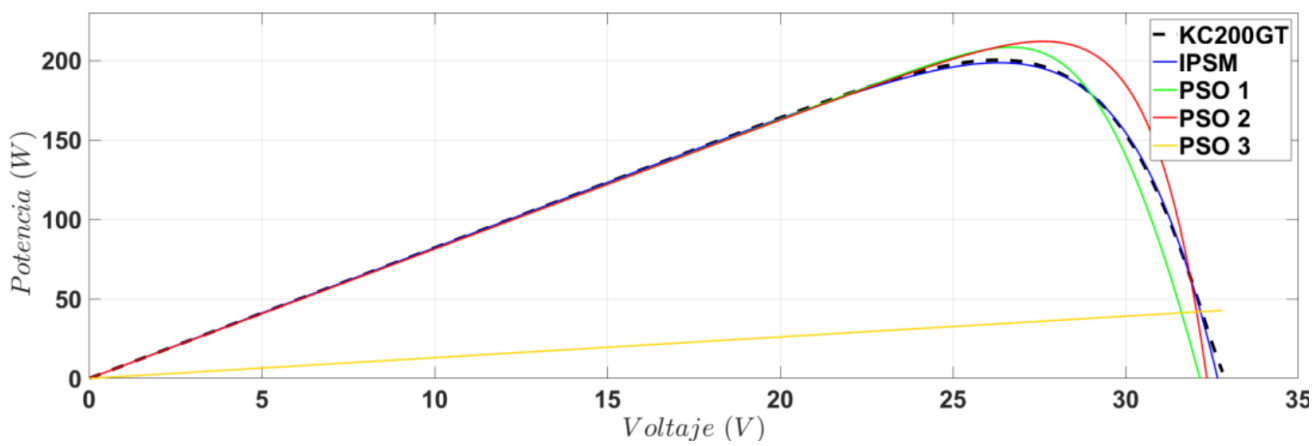

Figura 12. Comparación entre los datos de referencia del panel KC200GT, la estimación según IPSM y tres estimaciones según PSO Fuente: elaboración propia.

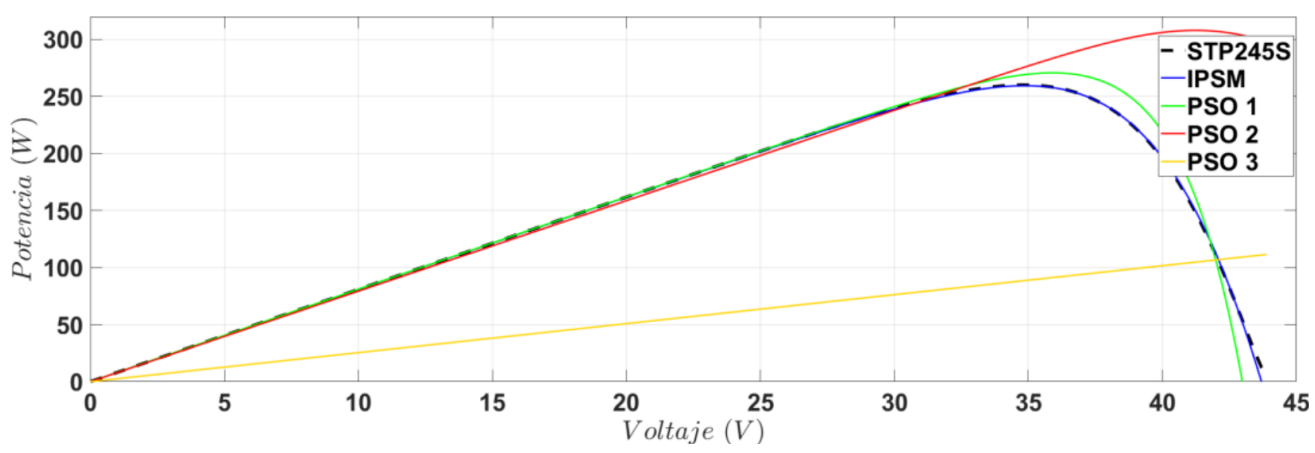

Figura 13. Comparación entre los datos de referencia del panel STP45S, la estimación según IPSM y tres estimaciones según PSO Fuente: elaboración propia. 
Como el algoritmo de optimización PSO depende de la aleatoriedad, se ejecutó tres veces para cada uno de los paneles. Por otro lado, el algoritmo de optimización IPSM solo se ejecutó una vez, ya que siempre arroja el mismo resultado. En la tabla 3 se pueden observar los parámetros obtenidos usando IPSM y que hacen que el modelo de un solo diodo se asemeje más a la curva de referencia se pueden observar.

Tabla 3. Mínimo global encontrado para $n, I_{0}, R_{s^{\prime}} R_{h^{\prime}} I_{p h}$ de cuatro paneles diferentes

\begin{tabular}{cccccc}
\hline Panel & $R_{h}(\Omega)$ & $R_{s}(\Omega)$ & $\eta$ & $I_{d 1}(A)$ & $I_{p h}(A)$ \\
\hline MSX60 & $9.502,56$ & 48,23 & 0,9915 & 3,7693 & 3,8002 \\
\hline JS65 & $3.591,17$ & 42,44 & 1,6744 & 5,0849 & 4,0046 \\
\hline KC200GT & $7,836,73$ & 48,23 & 1,0810 & 6,7323 & 8,2137 \\
\hline SPT245S & $3.915,08$ & 96,45 & 0,7608 & 2,4614 & 8,0941 \\
\hline
\end{tabular}

Fuente: elaboración propia.

La tabla 4 muestra el error absoluto de corriente, de acuerdo con la ecuación 11, para las estimaciones hechas para los cuatro paneles utilizando el algoritmo IPSM y el mejor caso del algoritmo PSO. Nótese que el error de la estimación realizada con el algoritmo IPSM siempre es menor que la obtenida en el mejor caso con el PSO. Por ejemplo, para el MSX60 el menor error obtenido con PSO es el 250,1 \% más que el error obtenido con el algoritmo IPSM. El caso en el cual las dos estimaciones tuvieron el error de corriente más semejante fue para la estimación PSO 1 (trazo verde en la figura 11) del panel JS65, donde el error con el PSO fue el 77,1 \% más del error obtenido con el algoritmo IPSM.

Sin embargo, cabe anotar que no todas las estimaciones con el algoritmo PSO convergen. Por ejemplo, la estimación PSO3 en los paneles JS65 series, KC200GT y STP245S muestran que el modelo no sigue a la referencia. Por ello, este algoritmo no garantiza, a diferencia del IPSM, la convergencia en todos los casos.

Finalmente, para obtener el modelo matemático de un diodo para cada uno de los cuatro paneles comerciales considerados en este trabajo, se debe reemplazar en la ecuación 2 los parámetros mostrados en la tabla 3.

Tabla 4. Error de corriente (ecuación 11) en las estimaciones

\begin{tabular}{cccc}
\hline Panel & $\operatorname{Error}_{I P S M}(A)$ & Error $_{P S O}(A)$ & Relación $^{(\%)}$ \\
\hline MSX60 & 1,0516 & 3,6813 & 250,1 \\
\hline JS65 & 3,0753 & 5,4471 & 77,1 \\
\hline KC200GT & 13,7053 & 46,5502 & 239,7 \\
\hline STP245S & 11,1318 & 42,8387 & 284,8 \\
\hline \multicolumn{2}{c}{ Fuente: elaboración propia. }
\end{tabular}




\section{CONCLUSIONES}

En este artículo se abordó la estimación de los parámetros del modelo matemático de un diodo para cuatro diferentes paneles fotovoltaicos de uso industrial: MSX60, JS65 Series, KC200GT y STP245S. Dicha estimación se realizó usando el algoritmo IPSM, el cual es una propuesta mejorada del conocido algoritmo PSM. Los parámetros del modelo que fueron estimados son: $R_{h}, R_{s}, \eta, I_{d 1}, I_{p h}$.

El modelo resultante obtenido a partir del algoritmo de optimización IPSM reproduce con buena exactitud la curva de referencia $V-P$ para los cuatro paneles. Se hicieron estimaciones utilizando el algoritmo de optimización de enjambre de partículas, $\mathrm{y}$ en todas las estimaciones los resultados obtenidos con el algoritmo IPSM fueron mejores que los otros algoritmos expuestos. Asimismo, se puede determinar que los resultados son un $50 \%$ mejores que los expuestos por PSM [41].

\section{REFERENCIAS}

[1] Congreso de la república, "Ley n. ${ }^{\circ} 1743 ”, 26$ de diciembre de 2014. Disponible: http:// wp.presidencia.gov.co/sitios/normativa/leyes/Documents/LEY\%201743\%20DEL\%2026\%20 DE\%20DICIEMBRE\%20DE\%202014.pdf

[2] N. Di, "Market Report Series energy efficiency 2017", 2017.

[3] E. E. Henao-Bravo and D. A. Márquez-Viloria, "Modelo matemático de sistemas fotovoltaicos para búsqueda distribuida del punto de máxima potencia Mathematical model of photovoltaic systems for distributed maximum power point tracking," Tecno Lógicas, vol. 19, n. ${ }^{\circ} 37$, pp. 107-124, 2016.

[4] D. J. Coyle et al., "LDi, “Market Report Series energy efficiency 2017”, 2017.ife prediction for CIGS solar modules part 2," Prog. Photovoltaics, pp. 156-172, 2013, https://doi.org/10.1002/ pip. 1171

[5] E. Skoplaki and J. A. Palyvos, "On the temperature dependence of photovoltaic module electrical performance: A review of efficiency/power correlations," Sol. Energy, vol. 83, n. ${ }^{\circ}$ 5, pp. 614-624, 2009, https://doi.org/10.1016/j.solener.2008.10.008

[6] E. Henao, D. Márquez, J. Villegas, S. Serna, C. Ramos, and D. González, “Modelo matemático de sistemas fotovoltaicos para búsqueda distribuida del punto de máxima potencia”, Tecno Lógicas, vol. 19, n. ${ }^{\circ}$ 37, pp. 108-124, 2016.

[7] J. D. Bastidas-Rodriguez, E. Franco, G. Petrone, C. A. Ramos-Paja, and G. Spagnuolo, "Model-Based Degradation Analysis of Photovoltaic Modules Through Series Resistance Estimation," IEEE Trans. Ind. Electron., vol. 62, n. ${ }^{\circ}$ 11, pp. 7256-7265, 2015, https://doi. org/10.1109/TIE.2015.2459380

[8] P. Bhatnagar and R. K. Nema, "Maximum power point tracking control techniques: Stateof-the-art in photovoltaic applications," Renew. Sustain. Energy Rev., vol. 23, pp. 224-241, 2013, https://doi.org/10.1016/j.rser.2013.02.011 
[9] D. González Montoya, "Control and optimization strategies to maximize the energy generated by photovoltaic sources" (Doctoral dissertation, Universidad Nacional de Colombia-Sede Manizales), 2017, http://www.bdigital.unal.edu.co/56931/

[10] G. Petrone, C. Ramos-Paja y G. Spagnuolo, "Photovoltaic Sources Modeling”. PV Models, 2017, https://www.researchgate.net/publication/319493486_Photovoltaic_Sources_Modeling

[11] X. H. Nguyen and M. P. Nguyen, "Mathematical modeling of photovoltaic cell/module/arrays with tags in Matlab/Simulink," Environ. Syst. Res., vol. 4, n. ${ }^{\circ}$ 1, 2015, https://doi.org/10.1186/ s40068-015-0047-9

[12] Pranahita, B. S., Kumar, A. S. y Babu, A. P. A. (2014). A Study on Modelling and Simulation of Photovoltaic Cells. Int J Res Eng Technol, 3, 101-8.

[13] H. Tian, F. Mancilla-David, K. Ellis, E. Muljadi, and P. Jenkins, "A cell-to-module-to-array detailed model for photovoltaic panels," Sol. Energy, vol. 86, n. ${ }^{\circ}$ 9, pp. 2695-2706, 2012, https://doi.org/10.1016/j.solener.2012.06.004

[14] P. Suskis and I. Galkin, "Enhanced photovoltaic panel model for MATLAB-simulink environment considering solar cell junction capacitance," Iecon Proc. (Industrial Electron. Conf., pp. 1613-1618, 2013, https://doi.org/10.1109/IECON.2013.6699374.

[15] T. Ahmad, S. Sobhan, and M. F. Nayan, "Comparative Analysis between Single Diode and Double Diode Model of PV Cell: Concentrate Different Parameters Effect on Its Efficiency," J. Power Energy Eng., vol. 04, n. ${ }^{\circ}$ 03, pp. 31-46, 2016, https://doi.org/10.4236/jpee.2016.43004.

[16] A. R. Jordehi, "Parameter estimation of solar photovoltaic (PV) cells: A review," Renew. Sustain. Energy Rev., vol. 61, pp. 354-371, 2016, https://doi.org/10.1016/j.rser.2016.03.049.

[17] O. Mares, M. Paulescu, and V. Badescu, "A simple but accurate procedure for solving the five-parameter model," Energy Convers. Manag., vol. 105, pp. 139-148, 2015, https://doi. org/10.1016/j.enconman.2015.07.046.

[18] J. Bai, S. Liu, Y. Hao, Z. Zhang, M. Jiang, and Y. Zhang, "Development of a new compound method to extract the five parameters of PV modules," Energy Convers. Manag., vol. 79, pp. 294-303, 2014, https://doi.org/10.1016/j.enconman.2013.12.041.

[19] L. Peng, Y. Sun, and Z. Meng, "An improved model and parameters extraction for photovoltaic cells using only three state points at standard test condition," J. Power Sources, vol. 248, pp. 621-631, 2014, https://doi.org/10.1016/j.jpowsour.2013.07.058.

[20] J. J. Soon, K. S. Low, and S. T. Goh, "Multi-dimension diode photovoltaic (PV) model for different PV cell technologies,” IEEE Int. Symp. Ind. Electron., pp. 2496-2501, 2014, https:// doi.org/10.1109/ISIE.2014.6865012.

[21] A. Orioli and A. Di Gangi, "A procedure to calculate the five-parameter model of crystalline silicon photovoltaic modules on the basis of the tabular performance data," Appl. Energy, vol. 102, pp. 1160-1177, 2013, https://doi.org/10.1016/j.apenergy.2012.06.036.

[22] A. Chouder, S. Silvestre, N. Sadaoui, and L. Rahmani, "Modeling and simulation of a grid connected PV system based on the evaluation of main PV module parameters," Simul. Model. Pract. Theory, vol. 20, n. ${ }^{\circ}$ 1, pp. 46-58, 2012, https://doi.org/10.1016/j.simpat.2011.08.011. 
[23] K. Ding, X. Bian, H. Liu, and T. Peng, "A MATLAB-simulink-based PV module model and its application under conditions of nonuniform irradiance," IEEE Trans. Energy Convers., vol. 27, n. ${ }^{\circ}$ 4, pp. 864-872, 2012, https://doi.org/10.1109/TEC.2012.2216529.

[24] V. Lo Brano, A. Orioli, G. Ciulla, and A. Di Gangi, “An improved five-parameter model for photovoltaic modules," Sol. Energy Mater. Sol. Cells, vol. 94, n. ${ }^{\circ}$ 8, pp. 1358-1370, 2010, https://doi.org/10.1016/j.solmat.2010.04.003.

[25] D. Oliva, E. Cuevas, and G. Pajares, "Parameter identification of solar cells using artificial bee colony optimization," Energy, vol. 72, pp. 93-102, 2014, https://doi.org/10.1016/j. energy.2014.05.011.

[26] A. Askarzadeh and A. Rezazadeh, "Artificial bee swarm optimization algorithm for parameters identification of solar cell models," Appl. Energy, vol. 102, pp. 943-949, 2013, https:// doi.org/10.1016/j.apenergy.2012.09.052.

[27] D. H. Muhsen, A. B. Ghazali, T. Khatib, and I. A. Abed, "Extraction of photovoltaic module model's parameters using an improved hybrid differential evolution/electromagnetism-like algorithm," Sol. Energy, vol. 119, pp. 286-297, 2015, https://doi.org/10.1016/j.solener.2015.07.008.

[28] L. L. Jiang, D. L. Maskell, and J. C. Patra, "Parameter estimation of solar cells and modules using an improved adaptive differential evolution algorithm," Appl. Energy, vol. 112, pp. 185-193, 2013, https://doi.org/10.1016/j.apenergy.2013.06.004.

[29] W. Gong and Z. Cai, "Parameter extraction of solar cell models using repaired adaptive differential evolution,” Sol. Energy, vol. 94, pp. 209-220, 2013, https://doi.org/10.1016/j. solener.2013.05.007.

[30] V. Khanna, B. K. Das, D. Bisht, Vandana, and P. K. Singh, "A three diode model for industrial solar cells and estimation of solar cell parameters using PSO algorithm," Renew. Energy, vol. 78, pp. 105-113, 2015, https://doi.org/10.1016/j.renene.2014.12.072.

[31] M. F. AlHajri, K. M. El-Naggar, M. R. AlRashidi, and A. K. Al-Othman, "Optimal extraction of solar cell parameters using pattern search,” Renew. Energy, vol. 44, pp. 238-245, 2012, https://doi.org/10.1016/j.renene.2012.01.082.

[32] K. M. El-Naggar, M. R. AlRashidi, M. F. AlHajri, and A. K. Al-Othman, "Simulated Annealing algorithm for photovoltaic parameters identification," Sol. Energy, vol. 86, n. ${ }^{\circ}$ 1, pp. 266-274, 2012, https://doi.org/10.1016/j.solener.2011.09.032.

[33] Q. Niu, L. Zhang, and K. Li, "A biogeography-based optimization algorithm with mutation strategies for model parameter estimation of solar and fuel cells," Energy Convers. Manag., vol. 86, pp. 1173-1185, 2014, https://doi.org/10.1016/j.enconman.2014.06.026.

[34] A. Askarzadeh and A. Rezazadeh, "Parameter identification for solar cell models using harmony search-based algorithms," Sol. Energy, vol. 86, n. ${ }^{\circ}$ 11, pp. 3241-3249, 2012, https:// doi.org/10.1016/j.solener.2012.08.018.

[35] V. J. Chin, Z. Salam, and K. Ishaque, "Cell modelling and model parameters estimation techniques for photovoltaic simulator application: A review," Appl. Energy, vol. 154, n. ${ }^{\circ}$, pp. 500-519, 2015, https://doi.org/10.1016/j.apenergy.2015.05.035. 
[36] J. D. Bastidas-Rodriguez, E. Franco, G. Petrone, C. A. Ramos-Paja, and G. Spagnuolo, "Quantification of photovoltaic module degradation using model based indicators," Math. Comput. Simul., vol. 131, pp. 101-113, 2017, https://doi.org/10.1016/j.matcom.2015.04.003.

[37] R. M. Corless, G. H. Gonnet, D. E. G. Hare, and D. E. Knuth, "On the Lambert W Function," D.E.G. al. Adv Comput Math, vol. 5, n. ${ }^{\circ}$ 5, pp. 329-330, 1996, https://doi.org/10.1007/ BF02124750.

[38] J. Accarino, G. Petrone, C. a. Ramos-Paja, and G. Spagnuolo, "Symbolic algebra for the calculation of the series and parallel resistances in PV module model," 4th Int. Conf. Clean Electr. Power Renew. Energy Resour. Impact, ICCEP, pp. 62-66, 2013, https://doi.org/10.1109/ ICCEP.2013.6586967.

[39] M. Clerc, "Standard Particle Swarm Optimisation: From 2006 to 2011," Preprint work document, 2012, hal-00764996.

[40] S. Lineykin, M. Averbukh, and A. Kuperman, "Five-parameter model of photovoltaic cell based on STC data and dimensionless," IEEE $27^{\text {th }}$ Conv. Electr. Electron. Eng. Isr., pp. 1-5, 2012, https://doi.org/10.1109/EEEI.2012.6377079.

[41] H. J. A. P. J. Tobon Andres Felipe, "Estimación de los parámetros de un modelo matemático de una celda fotovoltaica utilizando un algoritmo de optimización de búsqueda de patrones," Ingenio Magno, vol. 5, pp. 95-101, 2015, http://revistas.ustatunja.edu.co/index.php/ingeniomagno/article/view/883 